\section{Factors associated with stockout of Nevirapine syrup in Oshana Region, Namibia}

\author{
Emmanuel Magesa, \\ Kabwebwe Honore Mitonga, \\ Penehafo Angula \\ School of Public Health, University of \\ Namibia, Namibia
}

\begin{abstract}
Infants who are born with HIV/AIDS is still a public health concern in developing countries, especially in Sub Saharan Africa. In Namibia, it is estimated that the prevalence rate of HIV pregnant women is around $17.2 \%$, makes it to be among the countries with the highest prevalence rates among HIV women in sub-Saharan Africa (SSA). Some improvement regarding Prevention of Mother to Child Transmission has been made, however, frequent stock out of Nevirapine syrup continues to slow down, government efforts to foster the emergence of an HIV-free generation of Namibians. Given the nature of current conditions, justification exists for a study to determine the factors associated with stock out of Nevirapine (NVP) syrup. Mixed method was employed. In which descriptive approach was employed to acquire data from a period of five years retrospectively (2012-2016) and informants from different levels of supply chain in Oshana region. Initial, order receiving is not a predictor of NVP syrup. Lack of pharmaceutical knowledge is the biggest challenge which leads to sporadic stock out of NVP syrup. Pharmaceutical knowledge of ordering NVP syrup is needed to prevent stock out of NVP syrup. It is a recommendation of this study that training is needed for staffs who are involved in ordering of pharmaceutical items.
\end{abstract}

\section{Introduction}

Recently there has been much interest worldwide in the concept of supply chains of Anti Retroviral drugs (ARV) and its relevance. It is the main component of a systematic program for the treatment of HIV/AIDS. ${ }^{1}$ Supply chains of ARVs involve complex networks of heterogeneous stakeholders in both the public and private sectors, which include resources, technology, activities and information. These networks ensure that ARVs and related products, are moved from manufacturers to patients in an efficient manner. ${ }^{1-3}$
Strengthening of supply chain of ARVs, Nevirapine syrup in particular is very crucial in order to achieve a vision of Prevention of Mother to Child Transmission (PMTCT) by less than $5 \% .{ }^{1}$ In Namibia, several efforts have been done to strengthen supply chain of ARVs but frequency shortage and stock out has been reported. ${ }^{1,2}$ This situation may be caused by the increase of new HIV cases to the pregnant mothers, not attending or leave antenatal care (ANC) services, absence of a clear picture of aggregation of consumption figures of Nevirapine (NVP) syrup in medical stores and within health facilities, which has not been given much attention, fragmentation of supply chain in which ARVs subjected to different bodies of regulations, lack of good pharmaceutical knowledge for most of the health care workers at the facility level and long delivery lead time. ${ }^{4}$ This study has identified the factors associated with stock out and determine which factors can predict stock out of NVP syrup in public health facilities in Oshana region, Namibia.

A national cross sectional survey, which conducted in South Africa on Stock-outs of Antiretroviral ARV) which includes NVP syrup, indicated that lack of monitoring of stock is the main problem which leads to stock out. $^{5}$

Another cross sectional study supplemented with thematic approach was conducted in Tanzania regarding the stock out of ARVs revealed that inefficient supply systems, quantification problems and short expiry duration were cited as the main causes of stock-outs of ARVs. ${ }^{5,6}$ This study in Tanzania was also supported by another study done in Tehran, Iran which the finding indicated that. The major cause of shortage of medicine, particularly ARVs is lack of good forecasting consumption mechanism. ${ }^{4,7}$

A study conducted in Kenya and India to ascertain the factors influencing frequent stock-outs of essential medicines in public health facilities, reveals that $49.1 \%$ change on the availability of essential medicines could be attributed to independent variables. Information communication technology (ICT), lower lever of education was determined the most important factor, followed by inadequate staff qualifications, supply-chain design and monitoring and oversight mechanisms respectively. 6,8

Based on the literature review, the conceptual framework with main pillars which associated with stock out or shortage of NVP syrup in public health facilities was constructed. ${ }^{9}$

Figure 1, shows that overstock or understock is influenced by the pharmaceutical
Correspondence: Emmanuel Salvatory Magesa, School of Public Health, University of Namibia, Namibia

E-mail: emgesa2002@yahoo.com

Key words: Stock out, Nevirapine syrup, supply chain.

Acknowledgements: The authors wish to thank all staffs from Oshana health directorate who plays a key role in supply chain of essential medicines for their support with the study. No funding was received for the study. There is no conflict of interest in this study.

Contributions: The authors contributed equally

Conflict of interest: The authors declare no potential conflict of interest.

Funding: none.

Received for publication: 28 January 2019.

Revision received: 30 August 2019.

Accepted for publication: 17 October 2019.

This work is licensed under a Creative Commons Attribution NonCommercial 4.0 License (CC BY-NC 4.0).

(C) Copyright: the Author(s), 2019

Licensee PAGEPress, Italy

Journal of Public Health in Africa 2019; 10:1035 doi:10.4081/jphia.2019.1035

knowledge of health professionals, which in turn leads to stock out. Other factors in the conceptual framework are limited storage capacity, transportation and policy or guidelines Generally, stock out of NVP syrup is caused by different factors which link together as illustrated by Figure 1.

\section{Study design}

Mixed method, type of convergent parallel design was used. ${ }^{10}$ This means, both quantitative and qualitative data were collected at the same time. Start with retrospective, quantitative approach which collected data on NVP syrup for a period of 5 years (2012-2016) followed by qualitative approach, in which depth interview with informants focused on factors associated with stock out of NVP syrup in public health facilities in the region. The two methods were then compared at the interpretation stage. ${ }^{10}$

\section{Study setting}

The study was conducted in Oshakati Multi Regional Medical Depot (OMRMD) and all Primary Health Care (PHC) facilities in Oshana region except Eloolo clinic which start operation in 2015. 


\section{Informants}

Includes pharmacists, pharmacist assistants, nurses, transport officers and drivers who involve in supply chain in Oshana region.

\section{Sampling technique for NVP syrup}

Non probabilistic sampling method.

\section{Sampling technique for informants}

The researcher used, a stratified, purposive-sampling technique to identify health professionals and other professionals based on variation in experience and the kind of positions held, the researcher decided to use a purposive sampling technique known as maximum-variation sampling in order to tap the widest possible range of perspectives relating to the supply chain. ${ }^{11}$

\section{Sample size for key informants}

Saturation point was 17 informants.

\section{Data collection procedures}

Quantitative data on prevalence and incidence of HIV pregnant mothers was collected from District Health Information System (DHIS), consumption of nevirapine syrup was extracted from baby mother follow up reports, Electronic Dispensing Tools (EDT) and SYSPRO ${ }^{\circledR}$, nevirapine syrup register, inflation rates was extracted from economic monthly reports. Individuals face to face taped recorded interview with informants were conducted in order to gain more insight of the factors associated with stock out of Nevirapine syrup.

\section{Data analysis}

The data were then analyzed by SPSS version 24 software, in which Pearson correlation coefficient was used to find which factors are associated with stock out of NVP syrup and Binary logistic regression was applied to identify which factors associated with stock out of Nevirapine syrup can predict its stock out. Significant level set at $<0.05$. The qualitative aspects were analyzed thematically, whereby the main theme was stock out with seven sub themes.

\section{Ethical consideration}

The researcher sought permission from the University of Namibia (UNAM), School of Public Health, Ministry of Health and Social Services (MoHSS) and from respective study sites. No any name was identified or recorded.

\section{Results}

Binary logistic regression analysis was used to find out the factors associated with stock out of NVP syrup.

Table 1 showed that delivery lead time, initial stock of NVP syrup, number of HIV pregnant mothers prevalence and incidence of HIV pregnant mothers are statistically significant associated with stock out of NVP syrup. Table 2, show that delivery lead time is statistically significant predicts the stock out of NVP syrup in public health facilities at $\mathrm{P}$ value $<0.05$.

\section{Thematic analysis}

Theme 1. Stock out of NVP syrup. Sub theme 1a: Lack of consumption data

Most of informants, explain their experiences "The system which is in place is pull system, therefore We are assuming that what the health facility is requested is exactly what it need, but that is not the case, because most of health facilities, especially clinics are requesting more than or equivalent to a health centre or sometimes equivalent to hospital consumption!!. Therefore, based on the issuing alone does not reflect a true picture on the ground, though it is easy and not time consuming."

Sub theme 1b: Limited storage capacity

Informants at all levels stated that "Because of the limited storage and improper arrangement you will find that the medicines are ordered and receive, but don't use, but the staff realized that medicine was there, but have already gotten expired".

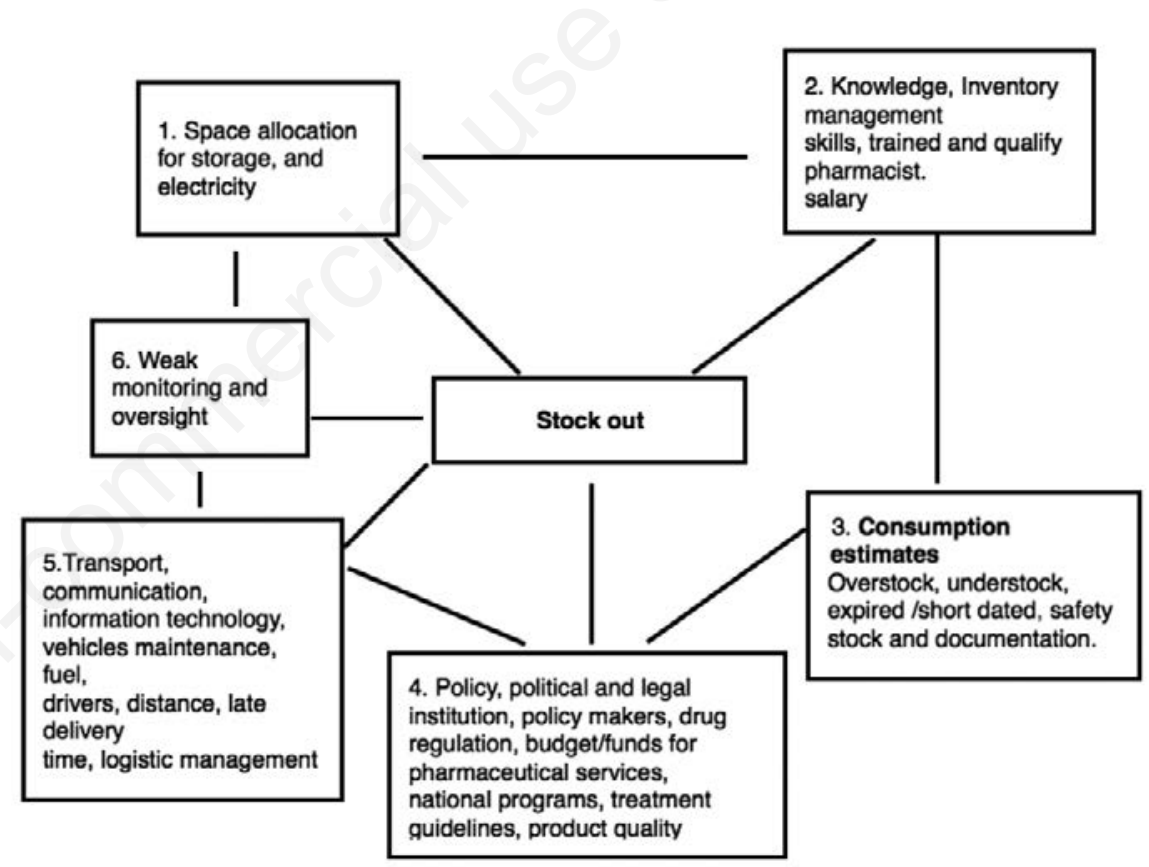

Figure 1. Conceptual framework of variables that causes stock out of mRDT in Oshana. Adapted and modified from the conceptual framework of Organizational practices influencing availability of essential medicines in hospitals.

Table 1. Factors associated with stock out of NVP syrup in public health facilities.

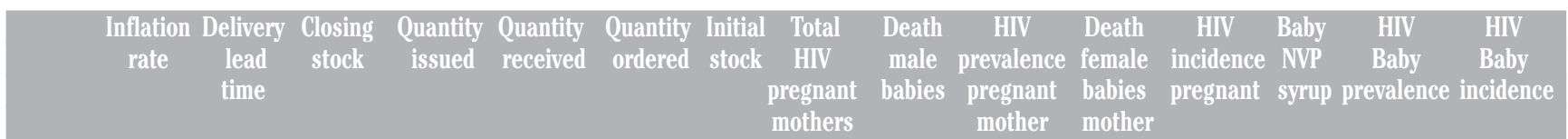

Pearson

\begin{tabular}{|c|c|c|c|c|c|c|c|c|c|c|c|c|c|c|c|}
\hline Correlation & .255 & .136 & -.323 & .220 & -.112 & -.146 & -.302 & -.341 & -.047 & -.332 & -.061 & .414 & -.193 & -.062 & -.053 \\
\hline Pvalue & .079 & $.003^{*}$ & .052 & .091 & .394 & .264 & .019 & .008 & .728 & .010 & .651 & .001 & .140 & .640 & .690 \\
\hline
\end{tabular}

*significant. 


\section{Sub theme 1c: Lack of transparency in pharmaceutical supply chain}

Informants said "Sometimes there is no communication between the management and other lower levels, e.g some time people from management level can come for supportive supervision once in a year. And most of the time we are not receiving any feedback/report after supervision".

\section{Sub theme 1d: Task shifting}

Informants had to say "Nurses have a lot of work to do relating to the nursing field, giving them another task (shifting task) will comprise the quality of services, and they will concentrate more on the task they know better".

Sub theme 1e: Shortage of trained staff

Some informants had the same view that the "Shortage of trained staff in the supply chain lead to overstock or understock especially at the lower level. Clinics are headed by registered nurses who are not well trained on the issue of supply chain of medicine".

\section{Sub theme 1f: Ordering and storage technique}

Informant said "We are ordering based on the ordering schedule which we received from OMRMD but the technique or what should we consider before ordering, we don't know!'”.

\section{Sub theme 1g: Delays in deliveries}

Informants from all levels of the supply chain were dissatisfied with the time of medicine delivery "There is no proper coordination between transport department and other departments dealing with pharmaceutical supply chain, this makes some delays to deliver medicines",

\section{Discussion}

In Table 1 coefficient correlation of Pearson indicates that, delivery lead time of NVP syrup, is associated with stock out of NVP syrup. This finding is the same from qualitative one, in which more informants mentioned the problem of delayed transport as the main issue which delay delivery of NVP syrup on time. All these findings from both methods are also supported by a study done in South Africa. ${ }^{12}$ Quantity ordered indicates by informants as one of the factors associated with stock out, however in quantitative findings, it indicates that it is not statistically significant. Some literatures are supporting that irregular ordering of medicines which mostly is due to lack of pharmaceutical skills, is associated with stock out. ${ }^{6}$ The increase of the number of HIV pregnant mothers is also found to be associated with stock out of NVP syrup. This might be true due to the increasing of consumption of NVP syrup because of the number of babies who are born with HIV. These reason is supported by the study on the challenges of stock out of ARV which was conducted in South Africa. $5,7,10,13$

From the findings, delivery lead time is found to be a predictor of stock out of NVP syrup in public health facilities in the region, This finding is also supported by several studies done in different parts of the words, indicating that lack of reliable transport makes the delayed of medicine delivery. $5,6,8$

\section{Conclusions}

Delivery lead time is the main factors and predictor of stock out of NVP syrup in public health facilities. Other factors also contribute, however many factors also relate to insufficient pharmaceutical knowledge. Strengthen transportation system and training health professionals, especially nurses would reduce the stock out of NVP syrup.

Table 2. Predictors of stock out of NVP syrup in public health facilities.

\begin{tabular}{|c|c|c|c|c|c|c|}
\hline $\begin{array}{l}\text { Variables in the Equation } \\
\text { Predictors }\end{array}$ & B & S.E. & Wald & df & Adjusted odds ratio, CI 95\% & $P$ value \\
\hline Delivery lead time & .049 & .002 & .889 & 1 & $1.050(.949-1.161)$ & $.036^{*}$ \\
\hline Initial stock & .004 & .003 & 1.650 & 1 & $1.004(.998-1.009)$ & .199 \\
\hline Total HIV pregnant mothers & .004 & .033 & .014 & 1 & $1.004(.942-1.070)$ & .906 \\
\hline HIV prevalence pregnant mother & .013 & 0.031 & 1.033 & 1 & $1.003(.9101-1.123)$ & .309 \\
\hline HIV incidence pregnant mother & .011 & 3.193 & 2.671 & 1 & $1.002(.353-1.037)$ & .102 \\
\hline
\end{tabular}

*significant.

Table 3. Integration between quantitative and qualitative findings.

\begin{tabular}{|c|c|c|c|}
\hline \multicolumn{2}{|c|}{ Quantitative } & \multicolumn{2}{|c|}{ Qualitative themes } \\
\hline Sources & Factors/variable & Stock out & Sources \\
\hline $\begin{array}{l}\text { SYSPRO, District Health } \\
\text { Information System (DHIS), }\end{array}$ & $\begin{array}{c}\text { Delivery lead time P value }<0.05 \\
\text { Total HIV pregnant mothers } \\
\text { Prevalence rate } \\
\text { Incidence rate }\end{array}$ & $\begin{array}{l}\text { Delayed delivery time. } \\
\text { Consumption increases due to } \\
\text { HIV new born babies. }\end{array}$ & Interview informants \\
\hline $\begin{array}{l}\text { Stock card, NVP register, } \\
\text { EDT, Baby mother follow up } \\
\text { reports }\end{array}$ & Initial stock & $\begin{array}{l}\text { "Transport is a challenge, } \\
\text { most of the time, we are receiving } \\
\text { NVP syrup beyond the prescribed time " } \\
\text { Irregular orders and poor documentation } \\
\text { Pharmaceutical knowledge }\end{array}$ & \\
\hline
\end{tabular}




\section{References}

1. IOM Institute of Medicine. Improving access to essential medicines for mental, neurological, and substance use disorders in sub-Saharan Africa: Workshop summary. Washington, DC: The National Academies Press; 2014.

2. World Health Organization. Operational Principles for Good Pharmaceutical Procurement, Geneva; 2012.

3. Spisak C, Morgan L, Eichler R, et al. Results-Based Financing in Mozambique's Central Medical Store: A Review After 1 Year. Global Health Sci Pract 2016;4:165-77.

4. Mokheseng M, Horn GA, Klopper GA. Supply chain solutions to improve the distribution of antiretroviral drugs (ARVs) to clinics in rural areas: A case study of QwaQwa districs.2017.

5. Bella H, Shroufi A, Gils T, et al. Stock- outs of antiretroviral and tuberculosis medicines in South Africa: A national cross-sectional survey. PLoS One 2019; 14:e0212405.

6. Mori AT, Owenya J. Stock-outs of antiretroviral drugs and coping strategies used to prevent changes in treatment regimens in Kinondoni District, Tanzania: a cross-sectional study. J Pharm Policy Pract 2014;7:3.

7. Gholami K, Kamalinia G, Ahmadian, MM, Salamzadeh J. Three Years Evaluation of Drug Shortages from Educational Pharmacies in Tehran. Iran J Pharmaceutic Res 2012;11:565-72.

8. Berhanemeskel E, Beedemariam G, Fenta GT., HIV/AIDS related commodities supply chain management in public health facilities of Addis Ababa, Ethiopia: a cross-sectional survey. J Pharm Pol Pract 2016:9-11.

9. Victor Z. Organizational practices influ- encing availability of essential medicines at hospitals in Nairobi county. 2017. Available from: https://pdfs. semanticscholar.org/b392/f0fcb5d6a11 e19c34ae8445a5234d06dff26.pdf.

10. Schoonenboom J, Johnson RB. How to Construct a Mixed Methods Research Design. Kolner Z Soz Sozpsychol 2017;69:107-31.

11. Guest G, Namey E. Public health research methods. London: SAGE Publications, Inc. 2015.

12. Bam L, McLaren ZM, Coetzee E, von Leipzig KH. Reducing stock-outs of essential tuberculosis medicines: a system dynamics modelling approach to supply chain management, Health Policy Plan 2017;32:1127-34.

13. Nana KP, Sandkjaer B. Meeting the Challenges to Scaling up HIV/AIDS Treatment in Africa. Dev Pract 2015;17: 272-84. 